\title{
Exploration and Practice of Solid State Physics Teaching Reform
}

\section{Zhonghui Xue}

School of Physics and Chemistry, Henan Polytechnic University, Jiaozuo, China

Email: hnlgxzh@163.com

How to cite this paper: Xue, Z.H. (2020) Exploration and Practice of Solid State Physics Teaching Reform. Open Access Library Journal, 7: e6004.

https://doi.org/10.4236/oalib.1106004

Received: December 16, 2019

Accepted: January 4, 2020

Published: January 7, 2020

Copyright (C) 2020 by author(s) and Open Access Library Inc.

This work is licensed under the Creative Commons Attribution International License (CC BY 4.0).

http://creativecommons.org/licenses/by/4.0/

\section{(c) (i) Open Access}

\begin{abstract}
Solid state physics is to study the structure and composition of the solid particle interaction and movement rules, and to elucidate the relationship between its structure and properties and its use of the subject, is the theoretical basis of new material technology. This paper, starting from solid state physics in the discipline of the importance and the current situation, combined with its own solid physics teaching practice, carried out some exploration on the teaching reform of solid state physics, to improve the quality of teaching of solid state physics course and expect that solid state physics teaching can keep up with the pace of the development of science and discipline.
\end{abstract}

\section{Subject Areas}

Classical Physics

\section{Keywords}

Solid Physics, Teaching Reform, Exploration, Creative Abilities

\section{1. 引言}

21 世纪, 随着科学技术的发展, 学科间的交叉融合越来越明显, 不同学 科间出现了越来越多的交集，科学逐渐向综合性方向发展[1]。固体物理作为 一门基础性学科, 受到了越来越多的重视。现在, 许多高等院校和科研机构 都开设了固体物理课。开设固体物理课的专业也由原来的物理学相关专业扩 展到了材料科学，光电子技术，微电子技术和能源技术等专业[2]。本文根据 应用物理类专业固体物理课程的开设情况与作者的实际教学经验, 对如何提 高应用物理专业固体物理教学质量, 促进学生综合能力的培养提出了一些自 己的浅见。 


\section{2. 固体物理教学的几点浅谈}

\section{1) 因地制宜制定选择教学内容是教学成功的关键}

学生的基础在一定程度上决定了教学内容的取舍, 在保证固体物理学基 本知识结构的基础上, 应当因地制宜的选择相应的章节进行教学实践。作为 我校本部学生来讲, 应用物理专业是二本学生, 学生的基础尚可, 可以适当 的选将叫固体物理学中较为抽象的内容进行课堂讲解, 而对于我校二级学院 - 万方科技学院的学生来讲, 是三本的学生, 学生的基础一般, 如果选择讲 授较为抽象的章节, 不但不能达到传授知识的目标, 而且会适得其反。

\section{2) 知识的融会贯通是培养能力的基础}

知识的传授永远都不是第一位的, 对大学生学习能力、应用能力和创新 能力的培养应当是高校专业教学的基本出发点和落脚点。知识的迁移能力是 融会贯通的基础。固体物理不同于普通物理学, 尽管应用物理专业的学生已 经学过了力、热、电、光、原, 然而固体物理的很多基本概念和基本知识却 是上面提到的五门课程中不曾涉及到的。尽管如此, 依然可以从普通物理学 中找到行对应的描述方法。比如我们在讲晶向指数和晶面指数时, 就可以借 用指点运动学中位置矢量的概念。而在讲授布拉菲晶格时, 我们可以借用质 点系的概念, 讲授格点时可以借用质点的概念, 从而达到知识的迁移和已有 知识和所讲知识的融会贯通。

\section{3) 研究型教学方法是培养创造性思维能力的切入点}

能力的培养和创造性思维方式的形成不是一朝一夕的事情, 作为高校老 师, 尤其是专业课教师, 在启迪和培养学生创造性思维能力和习惯方面就有 非常重要的作用。在固体物理某些章节的教学中, 我们采用三问: what, why, how 的方法开始某些章节的学习, 培养学生寻找、发现和解决问题的能力。

\section{4) 构建物理图像, 简化公式推导是教学成功的难点}

知识的记忆和理解, 图像的优势是显而易见。固体物理在有些知识点上 不像普通物理学, 学生较为容易构建起物理图像。作为讲授固体物理课程的 教师, 尤其是给应用物理学专业授课的教师, 就应当根据自己的经验和学识, 有意识的帮助学生构建物理过程, 形成物理图像, 而不能过分的强调公式的 推导。如在讲授能带论时, 引导学生从已学过的单原子分离能级出发, 不断 的在格点的位置上增加原子, 能级的交叠现象就会发生, 随着格点数目的增 多, 能级的交叠现象就越严重, 进而从一个量子化能级变成能带。

\section{5) 信息技术使用和网络资源的利用是提高学生学习质量的催化剂}

多媒体技术作为一种现代化的教学技术和工具, 将其应用到固体物理教 学中和课程实践中, 无疑能起到化抽象为生动的特殊作用; 借助网络环境, 对物理资源进行有机整合和优化, 扩展学生的知识面, 充分调动学生的积极 性, 达到提高学生学习兴趣, 提高学生探索问题、分析问题和解决问题的能 力。

对于原子密排形成的两种结构-六角密排和面心立方, 前者为六角密排结 构, 后者排列成面心立方结构, 而学生很难想象出后者怎么排列出来的。针 对这个难点, 利用多媒体动画, 就可以清楚地展示出原子排列结构如何从密 
排演变成面心立方的晶胞结构。推荐学生利用网络上一些免费软件建立三维 模型, 进而对模型进行任意角度旋转, 学生很快就掌握了, 教学效果很好。

\section{6) 强调网络资源的应用}

数字图书的使用。结合我校已有的数字资源情况, 以超星数字图书馆和 CNKI 中国学术期刊全文数据库为例, 给学生介绍如何利用网络资源。

课程网站的使用, 课件制作及收集。借鉴和吸收别人的长处, 参考其他 已有的资源和课件等, 进一步提高教学水平, 取得良好的教学效果。并给学 生介绍了如下几个学校的精品课程网站, 供其学习参考。

搜索网站的使用。如百度 https://www.baidu.com/, 谷歌 http://www.google.cn/的使用, 输入关键词, 可以得到需要检索的资料。网络 教学使教育的中心由教师转向学生, 学生是教学的主体, 教师的任务是要以 学生为中心, 激发学生获取知识的兴趣, 增进学生的求知欲, 并促使学生的 动力从外部转向内部、变被动学习为主动学习, 真正做到由 “要我学” 向 “我 要学” 的转换, 从而充分调动学生的积极性、创造性, 培养学生探索问题、 分析问题和解决问题的实际能力。

学术论坛的使用。如小木虫学术论坛, 星莹学术论坛, 诺贝尔学术论坛 等等。在这些论坛中有许多关于固体物理学习的讨论和资源。指导学生学会 通过和别人的交流讨论加深对固体物理研究内容的理解, 开拓思路。

\section{3. 结论}

在固体物理教学中采用多种方法和技术手段, 改革教学思想, 注重物理 思想、物理过程和物理图像的教学, 以能力为载体, 创新能力的培养为目标。 淡化公式推导, 变抽象内容灌输为物理图像再现, 使学生对事物有一个全面 准确的认识, 促进学生对知识的理解和巩固。同时借助网络环境, 对多种物 理资源进行有机整合, 深入推进教学改革。

\section{基金项目}

河南理工大学教学改革项目。

\section{References}

[1] 黄昆, 韩汝琦. 固体物理学 [M]. 北京: 高等教育出版社, 2005 重印.

[2] 曹全喜, 雷天民, 等. 固体物理基础 $[\mathrm{M}]$. 西安: 西安电子科技大学出版社, 2008. 